\title{
Possible Benefit of Dietary Carnosine towards Depressive Disorders
}

\author{
Alan R. Hipkiss* \\ School of Clinical and Experimental Medicine, College of Medical and Dental Sciences, University of \\ Birmingham, Edgbaston, Birmingham, B15 2TT, U.K.
}

[Received November 29, 2014; Revised December 10, 2014; Accepted December 11, 2014]

\begin{abstract}
Many stress-related and depressive disorders have been shown to be associated with one or more of the following; shortened telomeres, raised cortisol levels and increased susceptibility to age-related dysfunction. It is suggested here that insufficient availability of the neurological peptide, carnosine, may provide a biochemical link between stress- and depression-associated phenomena: there is evidence that carnosine can enhance cortisol metabolism, suppress telomere shortening and exert anti-aging activity in model systems. Dietary supplementation with carnosine has been shown to suppress stress in animals, and improve behaviour, cognition and well-being in human subjects. It is therefore proposed that the therapeutic potential of carnosine dietary supplementation towards stress-related and depressive disorders should be examined.
\end{abstract}

Key words: carnosine, telomeres, human, diet, supplementation, depression, cognition, stress, cortisol, aging

A recent paper [1] has shown that supplementation of human diets with carnosine ( $\beta$-alanyl-L-histidine), a naturally-occurring neurobiological peptide, can provoke beneficial effects on exercise performance and quality of life. Another recent paper [2] has shown that major depressive disorder (MDD) is associated with reduced telomere length, increased susceptibility towards agerelated dysfunction and raised cortisol levels in response to stress, while two other recent papers have described an association between decreased leukocyte telomere length and stress-associated disorders $[3,4]$. It is objective of the present communication to suggest that (i) carnosine insufficiency could provide a link between stress and depression-associated phenomena and age-related dysfunction, and (ii) carnosine may possess therapeutic potential towards depression and stress-associated disorders when administered as a dietary supplement.

\section{Carnosine and aging}

Carnosine is a normal constituent of brain and muscle. In the brain carnosine is synthesized by and secreted from astrocytes: it is degraded to its constituent amino acids by either cellular carnosinase (CNDP1) or serum carnosinase (CNDP2), whose activities in the brain possibly increase in old age [5]. Carnosine and related peptides such as anserine and balenine can also be obtained via carnivorous diets but are absent from strict vegetarian diets.

Although characterized more than a century ago [6], the "true" function of carnosine remains elusive, thus the dipeptide has been described as enigmatic [7]. Evidence from animal and model studies suggests that carnosine is multifunctional. Cell culture studies show that carnosine can inhibit growth of transformed cells [8-10], delay

*Correspondence should be addressed to: Dr. Alan R. Hipkiss, School of Clinical and Experimental Medicine, College of Medical and Dental Sciences, University of Birmingham, Birmingham, B15 2TT, U.K. Email: alanandjill@ lineone.net 
cellular senescence [11], help to maintain telomere length [12] and promote generation of a more juvenile phenotype when added to senescent cells [11]. Carnosine can inhibit many deleterious biochemical phenomena thought to be associated with generation of the aged phenotype, these include protein damage (including cross-linking [13]) induced by reactive oxygen species [14], reactive nitrogen species [15,16], reducing sugars [13] and reactive carbonyl species such as malondialdehyde [17], 4hydroxynonenal $[18,19]$ and methylglyoxal [20]. Model animal studies show beneficial effects of the dipeptide towards a number of age-related physiological conditions including impaired wound healing [21, 22], Alzheimer's disease [23, 24], Parkinson's disease [25, 26], stroke [27, 28], atherosclerosis [29, 30], cataracts [31, 32] and diabetic kidney disease [33, 34]. In fact it has been claimed that carnosine may be a rapamycin mimetic (a well-recognized anti-aging agent) because many of their respective properties are so similar [35], and recent evidence supports this proposal [10].

\section{Depression, cortisol, aging and carnosine}

The paper by Gotlib et al. [2] illustrates a relationship between telomere length, serum cortisol levels and depression in female children. It has also been suggested that major depressive disorder (MDD) patients may be predisposed to the onset of much age-related pathology [see 2 and refs. therein for a more complete discussion]. Papers by Miller and Sadeh [3] and Shaleh et al. [4] detail a relationship between telomere erosion and stress-related depressive disorders. The nature of the relationship, if any, between, on the one hand, cortisol levels and depression and, on the other hand, telomere length and aging, is uncertain. Nevertheless it is possible that a common molecular entity exists whose activities link aging, telomeres, cortisol and behaviour. It is suggested here that carnosine could provide a therapeutic link between these phenomena because, as noted above, carnosine (i) can act as an anti-aging agent (mimicking rapamycin) including exerting beneficial effects on animal models of age-related brain dysfunction [23-28], (ii) can help to maintain telomere length [12], (iii) may enhance cortisol metabolism, at least in mice [36], (iv) ameliorates stress-induced changes in metabolism in restrained mice [37] and (v), when complexed with zinc, suppress the effects of cortisol on rat bone metabolism [38]. Interestingly, it has been suggested that carnosine's anti-stress effects in mice are mediated by modulating the stress-activated hypothalamic-pituitary-adrenal axis [37], whilst it has recently been shown that raised cortisol levels are present in Alzheimer's disease patients' cerebrospinal fluid, possibly arising from dysregulation of the hypothalamic-pituitary-adrenal axis [39]. These observations indicate a possible role for carnosine in controlling age-related neurological dysfunction via effects on the hypothalamic-pituitary-adrenal axis in human brain.

\section{Carnosine supplementation affects brain function in chickens, rodents and humans}

Carnosine supplementation can influence behaviour in chickens [40] and rodents [41, 42]; its ability to suppress anxiety in rats [42] could possibly be due to enhanced cortisol metabolism [36]. Interestingly, it has also been reported that carnosine ( $\beta$-alanyl-histidine) and its reverse structure (i.e. histidinyl- $\beta$-alanine) exert the opposite behavioural effects in chickens [40, 43]. While the interpretation of this curious observation in very uncertain, it does at least indicate some structure-function relationship with respect to brain response to the peptides.

In humans, carnosine supplementation has been found to enhance cognition and/or well-being in at least five studies [1, 44-47]. Dietary supplementation with carnosine provoked beneficial effects on behaviour in autistic children [44], and improved cognitive function in patients suffering from Gulf War Illness [45], schizophrenia [46], and chronic heart failure [1]; the latter group also reported a significantly improvement in their quality of life [1]. A recent study has shown that carnosine plus anserine supplementation improved cognitive function and physical capacity in elderly humans [47]. These findings provide evidence that carnosine supplementation has therapeutic potential in humans, despite the presence of carnosinase which might be expected to counteract any efficacy that the dipeptide might elicit. It has however been shown that raised levels of kidney carnosinase does increase the probability of diabetic kidney disease in type-2 diabetics [34], which is probably due to increased destruction of the dipeptide. Nevertheless the fact that beneficial effects of carnosine supplementation have been detected in humans [1, 44-47] suggests that carnosinase activity is not necessarily an impediment to the dipeptide's efficacy in all cases.

\section{Conclusion}

Carnosine's ability to affect behaviour positively in humans when present as a dietary supplement supports the idea that carnosine should be explored for its therapeutic potential towards control of depression and post-traumatic stress disorder, as well as for the control of much agerelated pathology, despite of the presence of carnosinase. However it would be expected that geneticallydetermined differences in carnosinase activity could influence any beneficial effects of the dipeptide, as 
observed with respect to diabetic kidney disease [5, 33, 34].

\section{References}

[1] Lombardi C, Carubelli V, Lazzarini V, Vizzardi E, Bordonali T, Ciccarese C, Castrini AI, Dei Cas A, Nodari S, Metra M (2015). Effects of oral administration of orodispersible levo-carnosine on quality of life and exercise performance in patients with chronic heart failure. Nutrition, 31:72-8.

[2] Gotlib IH, LeMoult J, Colich NL, Foland-Ross LC, Hallmayer J, Joormann J, Lin J, Wolkowitz OM (2014). Telomere length and cortisol reactivity in children of depressed mothers. Mol Psychiatry, in press

[3] Miller MW, Sadeh N (2014). Traumatic stress, oxidative stress and post-traumatic stress disorder: neurodegeneration and the accelerated -aging hypothesis. Mol Psych, 19:1156-1162.

[4] Shalev I, Moffittt TE, Braithwaite AW, Danese A, Flemming NI et al. (2014). Internalizing disorders and leukocyte telomere erosion: a prospective study of depression, generalized anxiety disorder and posttraumatic stress disorder. Mol Psych, 19:1163-1169.

[5] Boldyrev, AA, Aldini, G, Derav W (2013). The physiology and pathophysiology of carnosine. Physiol. Rev, 93; 1803-1845.

[6] Skulachev VP (2000). Centenary of Gulevitsch's discovery. Biochem (Moscow), 65:749-750.

[7] Bauer K (2005). Carnosine and homocarnosine, the forgotten, enigmatic peptides of the brain. Neurochem Res, 30:1339-45.

[8] Holliday R, McFarland GA (1996). Inhibition of the growth of transformed and neoplastic cells by the dipeptide carnosine. Brit J Cancer, 73: 966-971.

[9] Gaunitz, F, Hipkiss AR (2012). Carnosine and cancer: a perspective. Amino Acids, 43:135-142.

[10] Zheng Z, Miao L, Wu X, Liu G, Peng Y, Xin X et al. (2014). Carnosine inhibits the proliferation of human gastric carcinoma cells by retarding Akt/mTOR/p70S6K signalling. J Cancer, 5:382-389.

[11] McFarland GA, Holliday R (1994). Retardation of senescence in cultured human diploid fibroblasts by carnosine. Exp Cell Res, 212: 167-175.

[12] Shao L, Li Q-H, Tan ZM (2004). L-Carnosine reduces telomere damage and shortening rate in cultured normal fibroblasts. Biochem Biophys Res Cmmns, 324: 931936.

[13] Hipkiss AR, Michaelis J, Syrris P (1995). Non-enzymic glycosylation of the dipeptide L-carnosine, a potential anti-protein-cross-linking agent. FEBS Letts, 371: 8185.

[14] Kohen R, Yamamoto Y, Cundy KC, Ames BN (1988). Antioxidant activity of carnosine, homocarnosine and anserine present in muscle and brain. Proc. Natl. Acad. Sci. USA, 95: 2175-2179.

[15] Fontana M, Pinnen F, Lucente G, Pecci L (2002). Prevention of peroxynitrate-dependent damage by carnosine and related sulphonamide pseudopeptides. Cell Mol Life Sci, 59: 546-55.

[16] Calabrese V, Coombrita C, Guagliano E, Sapoenza M, Ravagna A, Cardile V, et al. (2005). Protective effect of carnosine during nitrosactive stress in astroglial cell cultures. Neurochem Res, 30: 797-807.

[17] Hipkiss AR, Worthington VC, Himsworth, DT Herwig W (1998). Protective effects of carnosine against protein modification mediated by malondialdehyde and hypochlorite. Biochim Biophys Acta, 1380:46-54

[18] Orioli M, Aldini G, Beretta G, Mattei Facino R, Carini M (2005). LC-ESI-MS/MS determination of 4-hydroxytrans-2-nonenal Michael adducts with cysteine and histidine-containing peptides as early markers of oxidative stress in excitable tissues. J Chromatogr B Analyt Technol Biomed Life Sci, 827: 109-118.

[19] Liu Y, Xu G, Sayre LM (2003) Carnosine inhibits (E)-4hydroxy-2-nonenal-induced protein cross-linking: structural characterization of the carnosine-HNE adducts. Chem Res Toxicol, 16: 1589-1597.

[20] Hipkiss AR Chana, H (1998). Carnosine protects proteins against methylglyoxal-mediated modifications. Biochem Biophys Res Cmmns, 248:28-32.

[21] Nagai K, Suda T, Kawasaki K, Mathuura S (1986). Action of carnosine and $\beta$-alanine on wound healing. Surgery, 100: 815-821.

[22] Ansurudeen I, Sunkari VG, Grünler J, Peters V, Schmitt CP, Catrina SB, Brismar K, Forsberg EA (2012). Carnosine enhances diabetic wound healing in the $\mathrm{db} / \mathrm{db}$ mouse model of type 2 diabetes.Amino Acids, 43:12734.

[23] Herculano B, Tamura M, Ohba A, Shimatani M, Kutsuna $\mathrm{N}$, Hisatsune $\mathrm{T}$ (2013). $\beta$-alanyl-L-histidine rescues cognitive deficits caused by feeding a high fat diet in a transgenic mouse model of Alzheimer's disease. J Alzheimers Dis, 33:983-97.

[24] Corona C, Frazzini V, Silvestri E, Lattanzio R, La Sorda R, Piantelli M, Canzoniero LM, Ciavardelli D, Rizzarelli E, Sensi SL (2011). Effects of dietary supplementation of carnosine on mitochondrial dysfunction, amyloid pathology, and cognitive deficits in 3xTg-AD mice. PLoS One, 6(3):e17971.

[25] Afshin-Majd S, Khalili M, Roghani M, Mehranmehr N, Baluchnejadmojarad T (2014). Carnosine exerts neuroprotective effect against 6-hydroxydopamine toxicity in Hemiparkinsonian rat. Mol Neurobiol. In press

[26] Boldyrev AA, Stvolinsky SL, Fedorova TN, Suslina ZA (2010). Carnosine as a natural antioxidant and geroprotector: from molecular mechanisms to clinical trials. Rejuvenation Res, 13:156-8.

[27] Baek SH, Noh AR, Kim KA, Akram M, Shin YJ, Kim ES, Yu SW, Majid A, Bae ON (2014). Modulation of mitochondrial function and autophagy mediates carnosine neuroprotection against ischemic brain damage. Stroke, 45:2438-43.

[28] Wang JP, Yang ZT, Liu C, He YH, Zhao SS (2013). LCarnosine inhibits neuronal cell apoptosis through signal transducer and activator of transcription 3 signaling 
pathway after acute focal cerebral ischemia. Brain Res, 1507:125-33.

[29] Brown BE, Kim CH, Torpy FR, Bursill CA, McRobb LS, Heather AK, Davies MJ, van Reyk DM (2014). Supplementation with carnosine decreases plasma triglycerides and modulates atherosclerotic plaque composition in diabetic apo E(-/-) mice. Atherosclerosis, 232:403-9.

[30] Barski OA, Xie Z, Baba SP, Sithu SD, Agarwal A, Cai J, Bhatnagar A, Srivastava S (2013). Dietary carnosine prevents early atherosclerotic lesion formation in apolipoprotein E-null mice. Arterioscler Thromb Vasc Biol, 33:1162-70.

[31] Liao JH, Lin IL, Huang KF, Kuo PT, Wu SH, Wu TH (2014). Carnosine ameliorates lens protein turbidity formations by inhibiting calpain proteolysis and ultraviolet C-induced degradation.. J Agric Food Chem, 62:5932-8

[32] Dizhevskaya AK, Muranov KO, Boldyrev AA, Ostrovsky MA (2012). Natural dipeptides as minichaperones: molecular mechanism of inhibition of lens $\beta \mathrm{L}$-crystallin aggregation. Curr Aging Sci, 5:236-41.

[33] Aldini G, Orioli M, Rossoni G, Savi F, Braidotti P, Vistoli G, Yeum KJ, Negrisoli G, Carini M (2011). The carbonyl scavenger carnosine ameliorates dyslipidaemia and renal function in Zucker obese rats. J Cell Mol Med, 15:1339-54.

[34] Janssen B, Hohenadel D, Brinkkoetter P, Peters V, Rind $\mathrm{N}$, Fischer C, et al. (2005). Carnosine as a protective factor in diabetic nephropathy: association with a leucine repeat of the carnosinase gene CNDP1. Diabetes, 54:2320-7.

[35] Hipkiss, AR (2011). Energy metabolism, proteotoxic stress and age-related dysfunction - protection by carnosine. Mol. Aspects Med, 32:267-278.

[36] Nagai K, Suda T, Kawasaki K, Yamaguchi Y (1990). Acceleration of metabolism of stress-related substances by L-carnosine. Nihon Seirigaku Zasshi, 52:221-8.

[37] Tsoi B, He RR, Yang DH, Li YF, Li XD, Li WX, Abe $\mathrm{K}$, Kurihara H (2011). Carnosine ameliorates stressinduced glucose metabolism disorder in restrained mice. J Pharmacol Sci, 117:223-9.

[38] Segawa Y, Tsuzuike N, Tagashira E, Yamaguchi M. (1992). Beta-alanyl-L-histidino zinc prevents hydrocortisone-induced disorder of bone metabolism in rats. Res Exp Med (Berl), 192:317-22.
[39] Popp J, Wolfsgruber S, Heuser I, Peters O, Hull M, et al. (2014). Cerebrospinal fluid cortisol and clinical disease progression in MCI and dementia of Alzheimer's type. Neurobiol Aging, in press

[40] Tomonaga S, Tachibana T, Takagi T, Saito ES, Zhang R, Denbow DM, Furuse M (2004). Effect of central administration of carnosine and its constituents on behaviours in chicks. Brain Res Bull, 63:75-82.

[41] Li YF, He RR, Tsoi B, Li XD, Li WX, Abe K, Kurihara $\mathrm{H}$ (2012). Anti-stress effects of carnosine on restraintevoked immunocompromise in mice through spleen lymphocyte number maintenance. PLoS One, 7(4):e33190.

[42] Tomonaga S, Yamane H, Onitsuka E, Yamada S, Sato M, Takahata Y, Morimatsu F, Furuse M (2008). Carnosine-induced anti-depressant-like activity in rats. Pharmacol Biochem Behav, 89:627-32.

[43] Tsuneyoshi Y, Yamane H, Tomonaga S, Morishita K, Denbow DM, Furuse M (2008). Reverse structure of carnosine-induced sedative and hypnotic effects in the chick under acute stress. Life Sci, 82; 1065-1069

[44] Chez MG, Buchanan CP, Aimonovitch MC, Becker M, Schaefer K, Black C, Komen J (2002). Double-blind, placebo-controlled study of L-carnosine supplementation in children with autistic spectrum disorders. J Child Neurol, 17:833-7

[45] Baraniuk JN, El-Amin S, Corey R, Rayhan R, Timbol C (2013). Carnosine treatment for gulf war illness: a randomized controlled trial. Glob J Health Sci, 5:69-81.

[46] Chengappa KN, Turkin SR, DeSanti S, Bowie CR, Brar JS, Schlicht PJ, Murphy SL, Hetrick ML, Bilder R, Fleet D (2012). A preliminary, randomized, double-blind, placebo-controlled trial of L-carnosine to improve cognition in schizophrenia.. Schizophr Res, 142:145-52.

[47] Szczesniak D, Budzen S, Kopec W, Rymaszewska J (2014). Anserine and carnosine supplementation in the elderly: effects on cognitive functioning and physical capacity. Asch Gereontol Geriatr, 59:485-90. 